\title{
Does previous open surgical experience have any influence on robotic surgery simulation exercises?
}

\author{
Alin Adrian Cumpanas ${ }^{1}$, Razvan Bardan ${ }^{1}$, Ovidiu Catalin Ferician ${ }^{1}$, Silviu Constantin Latcu ${ }^{1}$, Ciprian Duta ${ }^{2}$, \\ Fulger Octavian Lazar ${ }^{2}$ \\ ${ }^{1}$ Department of Urology, Victor Babeș University of Medicine and Pharmacy, Timisoara, Romania \\ 2Department of Surgery, Victor Babeș University of Medicine and Pharmacy, Timisoara, Romania
}

Videosurgery Miniinv 2017; 12 (4): 366-371

DOI: https://doi.org/10.5114/wiitm.2017.72320

\begin{abstract}
Introduction: Within the last years, there has been a trend in many hospitals to switch their surgical activity from open/laparoscopic procedures to robotic surgery. Some open surgeons have been shifting their activity to robotic surgery. It is still unclear whether there is a transfer of open surgical skills to robotic ones.

Aim: To evaluate whether such transfer of skills occurs and to identify which specific skills are more significantly transferred from the operative table to the console.

Material and methods: Twenty-five volunteers were included in the study, divided into 2 groups: group A (15 participants) - medical students (without any surgical experience in open, laparoscopic or robotic surgery); and group $B$ (10 participants) - surgeons with exclusively open surgical experience, without any previous laparoscopic or robotic experience. Participants were asked to complete 3 robotic simulator console exercises structured from the easiest one (Peg Board) to the toughest one (Sponge Suture). Overall scores for each exercise as well as specific metrics were compared between the two groups.

Results: There were no significant differences between overall scores of the two groups for the easiest task. Overall scores were better for group $B$ as the exercises got more complex. For the intermediate and high-difficulty level exercises, most of the specific metrics were better for group $B$, with the exception of the working master space item. Conclusions: Our results suggest that the open surgical skills transfer to robotic skills, at least for the very beginning of the training process.
\end{abstract}

Key words: robotic surgery, open surgery, training simulator.

\section{Introduction}

The continuous worldwide expansion of robotic surgery forces surgeons to learn robotic procedures. With the exception of those having completed a formal robotic training program during their residency, the others have to "convert" from open/laparoscopic surgeons to console surgeons. The standard steps for initiation into robotic surgery include the online course (in order to get familiarized with the console, the commands, the docking process, trocar placement, etc), simulator exercises (on the da Vinci Skills Simulator), wet lab, viewing surgical procedures and proctor-supervised procedures. Until now, the majority of the studies related to the surgical skill transfer have addressed the laparoscopic surgeons versus surgical naïve medical students. There are studies suggesting that greater laparoscopic experience could lead to better results in difficult exercises, while having no impact on easy or intermediate 
tasks $[1,2]$. However, a small fraction of general surgeons, urologists or gynecologists are switching from exclusively open surgical experience to robotic surgery, and in these situations it is still unclear whether a transfer of surgical skills from open to robotic surgery exists. The clinical impact of our study is useful for that small fraction of surgeons directly converting from open to robotic surgery as, to our knowledge, until now there are no studies evaluating the transfer of surgical skills from open to robotic surgery.

\section{Aim}

The aim of our study was to evaluate whether the transfer of skills from open to robotic surgery occurs and to identify which specific skills are more significantly transferred from the operative table to the console. In other words, we aimed to answer the question: Do open surgeons complete the learning curve faster than open surgery-naïve trainees?

\section{Material and methods}

In this prospective study, we enrolled 25 voluntary robotic surgery trainees (without any robotic surgery experience or training), who had to complete three virtual reality exercises included in the da Vinci Skills Simulator (dVSS) program for training in robotic surgery (dV-Trainer, Mimic Technologies, Seattle, WA, USA). They were divided into two groups: group A 15 trainees without any surgical experience (medical students in their final year of study) and group $B$ - 10 trainees - surgeons with exclusively open surgical experience of more than 2 years and without any laparoscopic or robotic surgery experience. To avoid biases, previous video gaming of more than a total lifetime of $10 \mathrm{~h}$ represented an exclusion criterion. The study was conducted according to the hospital ethical requirements and consent was obtained from the participants of the study.

Three simulator exercises with increasing degrees of difficulty were selected from the virtual reality platform of the da Vinci Xi system: Peg Board level 1 exercise 1 (the easiest task); Energy Dissection level 2 - exercise 2 (intermediate task); and, finally, Suture Sponge level 3 - exercise 3 - the most difficult task and reported in the literature as one of the most reliable exercises to reveal the difference between beginners, intermediates and experts in robotic surgery [1].

Each trainee had the task explained to them before practicing simulator exercises as well as hav- ing completed the Da Vinci online training for basic knowledge of robot commands and functions (www. davincisurgerycommunity.com). Before starting the exercises and measuring the results, each trainee had a warm-up of 5 min for each exercise, to get to know the commands and the exercise requirements. Each exercise was performed three times, the mean value being considered. No feedback or instructions were given to the participants during the exercises.

The goal of the Peg Board exercise was to place the highlighted ring on the highlighted peg on the floor using two needle drivers. The Energy Dissection task was to sever each smaller vessel by applying bipolar energy at two points, then cutting in between. The Suture Sponge task consisted in passing a curved needle through designated dots on a sponge, from different angles, by a forehand or backhand approach. All these exercises were quantitatively evaluated by the simulator's software and the results between two groups were compared.

The overall score (the percentage displayed at the end of each exercise) as well as metrics specific to a certain exercise or common to two or three exercises, such as time to complete (seconds), object drop, instrument out of view, the economy of motion (the distance covered by the instruments to accomplish the task in $\mathrm{cm}$ ), the collision of the instruments, misapplied energy time, excessive instrument force and master workspace (the diameter of the working space on master grips in centimeters), were used to compare the two groups. Mean values were calculated after three trials of the same exercise type performed by each study participant.

\section{Statistical analysis}

Statistical analysis was performed, comparing the two groups, using the two-tailed paired $t$ test. A $p$-value $<0.05$ was considered significant.

\section{Results}

The mean age in group A was 22 years (range: 20-26) while in group B it was 38 years (range: 30-54). The majority of the participants were males $(10 / 15(66 \%)$ in group A and $8 / 10(80 \%)$ in group B) and right-handed (14/15 (93.3\%) in group $A$ and 10/10 (100\%) in group B) (Table I).

For exercise 1 (Peg Board 1) the overall score difference between the two groups was not statistically 
Table I. Demographic characteristics of the two groups

\begin{tabular}{|lccc|}
\hline Parameter & $\begin{array}{c}\text { Group A } \\
\text { (medical } \\
\text { students) } \\
n=15\end{array}$ & $\begin{array}{c}\text { Group B } \\
\text { (open } \\
\text { surgeons) } \\
n=10\end{array}$ & $P$-value \\
\hline Age & $22(20-26)$ & $38(30-54)$ & 0.1 \\
\hline Male/female & $10 / 5(66 \%)$ & $8 / 2(80 \%)$ & 0.2 \\
\hline Right handed & $14(93.3 \%)$ & $10(100 \%)$ & 0.2 \\
\hline
\end{tabular}

significant, being $78 \%$ in group $A$ and $80 \%$ in group $B$ $(p=0.5)$. For exercise 2 (Energy Dissection level 2), the overall score was $60 \%$ for group $A$ and $68 \%$ for group B $(p<0.05)$. For exercise 3 (Suture Sponge level 3 ), the overall score was $42 \%$ for group $A$ and $59 \%$ for group B $(p<0.05)$.
For the first exercise (Peg Board 1) we found statistically significantly better results in group B vs. group A for drops and the economy of motion ( $p<$ 0.05 for each of them). There were no differences regarding the instruments out of view, instrument collision, excessive force or time to complete the exercise $(p>0.05, p=0.5, p>0.05$ and $p=0.5$ respectively). The master workspace range was significantly better for group A vs. group B $(p<0.05)$ (Table II).

For exercise 2 (Energy Dissection level 2) statistically significantly better results were noted for the following items: time to complete, economy of motion, instrument collision, excessive force, instrument out of view ( $p<0.05$ for all of them). The master workspace range was, as in exercise 1 , significantly better for group A $(p<0.05)$ (Table III).

Table II. Overall results and specific metrics for exercise 1 - Peg Board level 1. Bold values indicate statistically significant differences and in favor of which group

\begin{tabular}{|lccc|}
\hline Parameter & $\begin{array}{c}\text { Group A (medical students) } \\
n=15 \\
\text { Mean }(95 \% \mathrm{Cl})\end{array}$ & $\begin{array}{c}\text { Group B (open surgeons) } \\
n=10 \\
\text { Mean }(95 \% \mathrm{Cl})\end{array}$ & \begin{tabular}{c}
$P$-value \\
\hline Overall score
\end{tabular} \\
\hline Time to complete $[\mathrm{s}]$ & $95(71-84)$ & $96(77-82)$ & 0.5 \\
\hline Instrument collision & $0.92(0.5-0.98)$ & $0.94(0.8-0.95)$ & 0.2 \\
\hline Excessive force & $0.1(-0.1-0.3)$ & $0.07(-0.2-0.18)$ & 0.5 \\
\hline Instruments out of view & $0.32(-0.15-0.52)$ & $0.40(-0.03-0.47)$ & 0.25 \\
\hline Economy of motion $[\mathrm{cm}]$ & $167(162-171)$ & $\mathbf{1 4 5}(135-148)$ & 0.85 \\
\hline Drops & $0.66(0.01-0.72)$ & $\mathbf{0 . 1 8}(0.03-0.5)$ & $\mathbf{0 . 0 1 2}$ \\
\hline Master workspace & $\mathbf{9 ( 7 - 1 1 )}$ & $11(9-15)$ & $\mathbf{0 . 0 1 3}$ \\
\hline
\end{tabular}

Table III. Overall results and specific metrics for exercise 2 - Energy Dissection level 2. Bold values indicate statistically significant differences and in favor of which group

\begin{tabular}{|c|c|c|c|}
\hline Parameter & $\begin{array}{c}\text { Group A (medical students) } \\
n=15 \\
\text { Mean }(95 \% \mathrm{Cl})\end{array}$ & $\begin{array}{c}\text { Group B (open surgeons) } \\
n=10 \\
\text { Mean }(95 \% \mathrm{Cl})\end{array}$ & $P$-value \\
\hline Overall score & $60(58-62)$ & $68(65-75)$ & 0.025 \\
\hline Time to complete [s] & 115 (107-124) & $103(91-115)$ & 0.018 \\
\hline Economy of motion [cm] & $190(180-195)$ & $161(149-164)$ & 0.014 \\
\hline Instrument collision & $0.5(-0.03-0.7)$ & $0.1(-0.01-0.3)$ & 0.03 \\
\hline Excessive force & $1.2(-0.98-2.3)$ & $0.32(-0.79-1.01)$ & 0.018 \\
\hline Instruments out of view & $1.1(-0.1-1.9)$ & $0.2(-0.1-0.8)$ & 0.01 \\
\hline Misapplied energy time & $7.2(6.9-8.4)$ & $6.7(5.9-7.5)$ & 0.024 \\
\hline Master workspace & $15(13-18)$ & $16.3(13-20)$ & 0.03 \\
\hline
\end{tabular}


Table IV. Overall results and specific metrics for exercise 3 - Suture Sponge level 3. Bold values indicate statistically significant differences and in favor of which group

\begin{tabular}{|c|c|c|c|}
\hline Parameter & $\begin{array}{c}\text { Group A (medical students) } \\
n=15 \\
\text { Mean }(95 \% \mathrm{Cl})\end{array}$ & $\begin{array}{c}\text { Group B (open surgeons) } \\
n=10 \\
\text { Mean }(95 \% \mathrm{Cl})\end{array}$ & $P$-value \\
\hline Overall score & $42(40-44)$ & $59(56-64)$ & 0.02 \\
\hline Time to complete [s] & $475(438-494)$ & 265 (259-269) & 0.015 \\
\hline Economy of motion $[\mathrm{cm}]$ & $598(578-600)$ & $375(356-380)$ & 0.025 \\
\hline Instrument collision & $7.3(5.8-7.6)$ & $2.7(2.4-3.4)$ & 0.01 \\
\hline Excessive force & $0.65(-0.15-0.95)$ & $0.2(-0.1-0.5)$ & 0.02 \\
\hline Instruments out of view & $1.35(1-2.2)$ & $0.98(0.79-1.21)$ & 0.025 \\
\hline Drops & $0.9(0.3-1.4)$ & $0.2(0.03-0.7)$ & 0.01 \\
\hline Master workspace & $8.4(7-9.2)$ & $8.1(7.4-9.5)$ & 0.3 \\
\hline
\end{tabular}

For exercise 3 (Suture Sponge level 3), statistically significantly better results were noted in group $B$ for all items (time to complete, economy of motion, instrument collision, excessive force, instruments out of view and drops $p<0.05$ for each of them), except the master workspace range, which had no statistical difference between the two groups $(p=0.3)$ (Table IV).

\section{Discussion}

As robotic surgery tends to replace open and laparoscopic procedures in urology, there are many urologists who would have to switch directly from the open to the robotic approach. Thus, a question arises: are open and robotic surgery skills interchangeable? If so, to what extent? It is hard to answer this question. The only reasonably objective tool would be the use of virtual reality simulators, to assess the robotic skills and to compare them with those of open surgical naive console surgeons. Mimic dV-Trainer was the first commercially available simulator for robotic training in 2007, and its newest version has been available on the Xi platform since 2014, being independently validated as face, content, construction, concurrency and prediction. It offers more than 60 exercises which allow the evaluation and development of different skills (e.g. camera control, use of instruments, EndoWrist manipulation, etc.) [3-5].

In the pursuit of fulfilling the aim or our study, we carefully defined the inclusion criteria in order to avoid biases and meticulously ensured proper selection of exercises and data analysis methods.
Inclusion criteria were very important since they represent bias factors that can easily alter the results. There are studies published in the literature regarding the impact of open surgical experience on robotic surgery but in those studies the robotic-naïve surgeons have had a wide range laparoscopic experience, biasing the results [6]. Although data from the literature suggest that at a group level there is no significant transfer of laparoscopic to robotic skills $[1,2]$, differences may occur due to the surgeon's experience in laparoscopic surgery [7]. For this reason, we formed the control group from medical students in their final year of medical school, without any surgical experience, as Hung et al. did for the validation of the first da Vinci Skills Simulator [8]. On the other hand, as there are conflicting data regarding the impact of video gaming experience in robotic surgery skills [9] we did not included in the study groups trainees with a lifetime video gaming experience of more than $10 \mathrm{~h}$.

The overall score, time to complete, economy of motion, instrument collision and master workspace were described as the most reliable metrics for the tasks on the dVSS [7]. However, it is still unclear whether these metrics are derived from the expert performance [6]. Our results revealed that the difference between the overall scores was not statistically significant between the two groups for exercise 1 (Peg Board), probably due to the lack of difficulty, the exercise being one of the easiest, assessing the EndoWrist manipulation and the hand-eye coordination. Although there is not a statistically significant difference in overall score between the two groups, 
one can see that the medical students perform better on simple tasks. Thus, for simple tasks, the surgical experience does not have an immediate impact on robotic skills. However, considering specific metrics of exercise 1 as drops and economy of motion, the results were better for experienced surgeons, whilst medical students better managed the master workspace, probably due to the tendency of the surgeons group to replicate their hand movements from open surgery to the console.

As the difficulty of the exercises increased (Energy Dissection level 2 and Sponge Suture level 3) the overall score difference, as well as time to complete, economy of motion, excessive force, instrument out of view and instrument collision, was statistically significantly in favor of the open surgeons group. Beside the EndoWrist manipulation and eye-hand coordination on the console (which get better results for group A), the experience in proper cutting, enough coagulation and the skill set of driving the needle from different angles for suturing makes the difference for open surgeons. The study of Finnerty et al. draws a similar conclusion when comparing medical students with general surgery residents with laparoscopic experience but, at the same time, it was observed that greater laparoscopic experience did not necessarily transfer to better robotic simulator results [1].

Interestingly enough, the master workspace as an individual metric remained better for medical students during exercise 2 (thus, they better managed their workspace during the exercise, although scoring poorly). As the trial gets more complex and they reach exercise 3 , they continue to have low scores, but, surprisingly, show a previously unseen poor workspace management. As a result, regarding the Sponge Suture exercise, there was no statistically significant difference in mastering the workspace between the two groups. This means that the lack of experience in handling and driving the curved needle (using the forehand and the backhand to make the suture) leads to the expansion of the workspace as the difficulty of the task increases. However, the overall average scores for all the three exercises in both groups suggests that systematic training in robotic surgery is mandatory to achieve satisfactory skills and to achieve proficiency.

While our study brings reliable data related to the transfer of the open surgical skills into robotic skills by trying to avoid biases, the major limitation consists in the small sample size and the limited number of exercises. The first aspect is related to the number of available volunteers who fulfilled the inclusion criteria, it being hard to find open surgeons without any experience in laparoscopy. We designed the study with only three exercises because we preferred to select those which could better evaluate the skills in robotics $[1,7]$ in a single session, without any biases which could result from consecutive days of practice. Meanwhile, we designed the study with only three trials for each exercise to evaluate the translation of the skills only, without any interference of the learning curve, reported to be reached on simulators after six attempts. On the other hand, it is unclear how the skills achieved through simulation training will translate to real-life surgery, although we can presume that previous surgical experience, knowledge of the local anatomy, of the cleavage plans and approaches to a certain area, etc., would be useful for the conversion of the open surgeon to robotic surgery. Future studies might be useful for more consistent data and, furthermore, for the evaluation of in-vivo results.

\section{Conclusions}

Our results suggest that open surgical skills transfer to robotic skills. The more complex the task, the more visible is the difference between the novices and experienced open surgeons. Although the results reveal the differences only for the very beginning steps of the training process, it can be supposed that previous experience in open surgery would be useful in real-life robotic surgical procedures.

\section{Conflict of interest}

The authors declare no conflict of interest.

\section{References}

1. Finnerty BM, Afaneh C, Aronova A, et al. General surgery training and robotics: are residents improving their skills? Surg Endosc 2016; 30: 567-73.

2. Panait L, Shetty S, Shewokis PA, Sanchez JA. Do laparoscopic skills transfer to robotic surgery? J Surg Res 2014; 187: 53-8.

3. Perrenot C, Perez M, Tran N, et al. The virtual reality simulator $\mathrm{dV}$-Trainer is a valid assessment tool for robotic surgical skills. Surg Endosc 2012; 26: 2587-93.

4. Kelly DC, Margules AC, Kundavaram CR, et al. Face, content, and construct validation of the da Vinci Skills Simulator. Urology 2012; 79: 1068-72.

5. Finnegan KT, Meraney AM, Staff I, Shichman SJ. da Vinci Skills Simulator Construct Validation Study: correlation of prior ro- 
botic experience with overall score and time score simulator performance. Urology 2012; 80: 330-6.

6. Gomez PP, Willis RE, Van Sickle KR. Development of a virtual reality robotic surgical curriculum using the da Vinci Si surgical system. Surg Endosc 2015; 29: 2171-9.

7. Lyons C, Goldfarb D, Jones SL, et al. Which skills really matter? Proving face, content, and construct validity for a commercial robotic simulator. Surg Endosc 2013; 27: 2020-30.

8. Hung AJ, Zehnder P, Mukul Patil, et al. Face, content and construct validity of a novel robotic surgery simulator. J Urol 2011; 186: 1019-25.

9. Lynch J, Aughwane P, Hammond TM. Video games and surgical ability: a literature review. J Surg Educ 2010; 67: 184-9.

Received: 5.09.2017, accepted: 17.10.2017. 